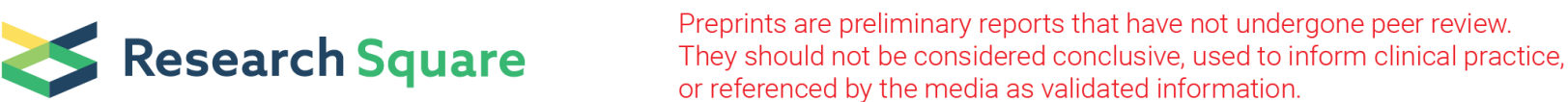

\section{Sex-Specific Alterations in Inflammatory MicroRNAs in Mouse Brain and Bone Marrow CD11b+ Cells Following Traumatic Brain Injury}

\section{Paresh Prajapati}

University of Kentucky College of Medicine

Wang-Xia Wang ( $\sim$ wwangc@uky.edu )

University of Kentucky College of Medicine https://orcid.org/0000-0002-8104-3779

\section{Steven A. Pesina}

University of Kentucky College of Medicine

\section{Urim Geleta}

University of Kentucky College of Medicine

Joe E. Springer

University of Kentucky College of Medicine

\section{Short Report}

Keywords: MicroRNAs, Traumatic brain injury (TBI), Inflammation, CD11b, Sexual dimorphism, Bone marrow

Posted Date: September 17th, 2021

DOI: https://doi.org/10.21203/rs.3.rs-899711/v1

License: (c) (1) This work is licensed under a Creative Commons Attribution 4.0 International License. Read Full License

Version of Record: A version of this preprint was published at Cellular and Molecular Neurobiology on November 10th, 2021. See the published version at https://doi.org/10.1007/s10571-021-01164-6. 


\section{Abstract}

Sex is a key biological variable in traumatic brain injury (TBI) and plays a significant role in neuroinflammatory responses. However, the molecular mechanisms contributing to this sexually dimorphic neuroinflammatory response remain elusive. Here we describe a significant and previously unreported tissue enrichment and sex specific alteration of a set of inflammatory microRNAs (miRNAs) in $\mathrm{CD} 11 \mathrm{~b}+$ cells of brain and bone marrow isolated from naïve mice as well as mice subjected to TBI. Our data from naïve mice demonstrated that expression levels of miR-146a-5p and miR-150-5p were relatively higher in brain CD11b + cells, and that miR-155-5p and miR-223-3p were highly enriched in bone marrow CD11b + cells. Furthermore, while miR-150-5p and miR-155-5p levels were higher in male brain CD11b + cells, no significant sexual difference was observed for miR-146a-5p and miR-223-3p. However, TBI resulted in sex specific differential responses of these miRNAs in brain CD11b + cells. Specifically, miR223 levels in brain CD11b + cells were markedly elevated in both sexes in response to TBI at 3 and $24 \mathrm{hr}$, with levels in females being significantly higher than males at $24 \mathrm{hr}$. We then focused on analyzing several miR-223-3p targets and inflammation-related marker genes following injury. Corresponding to the greater elevation of miR-223-3p in females, the miR-223-3p targets, TRAF6 and FBXW7 were significantly reduced in females compared to males. Interestingly, anti-inflammatory genes $A R G 1$ and IL 4 were higher in females after TBI than in males. These observations suggest miR-223-3p and other inflammatory responsive miRNAs may play a key role in sex-specific neuroinflammatory response following TBI.

\section{Introduction}

Neuroinflammation is recognized as a critical factor to acute and chronic secondary injury following traumatic brain injury (TBI). While the acute inflammatory stage is essential for removal of cellular debris and promoting cellular repair, dysregulation of this response leads to chronic microglia activation and pro-inflammatory signaling impacting long-term neurological function (Johnson et al. 2013; Loane et al. 2014; Pierce et al. 1998). This is characterized by the excessive release of pro-inflammatory factors by resident and recruited myeloid cells that differentiate along a continuum from cytotoxic to reparative phenotypes (Jin et al. 2012; Kumar et al. 2016; Morganti et al. 2016). This neuroinflammatory response also is sex-specific and characterized by a differential myeloid cell response in females relative to males (Acaz-Fonseca et al. 2015; Doran et al. 2019; Villapol et al. 2017).

MicroRNAs (miRNAs) are non-coding RNAs that regulate gene expression post-transcriptionally in almost all cellular events, including those controlling sex-specific responses (Kodama et al. 2020; Liu and Xu 2011; Sharma and Eghbali 2014). Numerous studies including ours have reported a strong correlation between altered miRNA expression levels and secondary brain injury events (Liu et al. 2014; Redell et al. 2009; Wang et al. 2015). Among these altered miRNAs, miR-146a, miR-223-3p, miR-155-5p, and miR-150 play key roles in inflammatory signaling (Cardoso et al. 2012; Zhao et al. 2011; Johnnidis et al. 2008; Shakerian et al. 2018). However, a mechanistic role for these inflammatory miRNAs in TBI-induced neuroinflammation is poorly understood. Moreover, no studies have revealed the involvement of these miRNAs to sex-specific neuroinflammatory responses. 
Cluster of differentiation molecule 11b (CD11b, or Integrin alpha M (ITGAM)) is a leukocyte surface marker highly expressed on monocytes and macrophages of bone marrow as well as on resident CNS microglia (Gordon and Taylor 2005). Here we report a differential enrichment of these miRNAs in mouse CD11b + cells from bone marrow and brain, and a time-dependent alteration of these miRNAs following TBI. Our study suggests that the enrichment and alteration of miRNAs in CD11b + cells of a particular tissue origin may reflect their specific immunomodulatory role in response to TBI.

\section{Materials And Methods}

\section{Animal Studies: Controlled Cortical Impact Injury}

All animal methodology was in full compliance with the National Institutes of Health (NIH) Guide and with the US Public Health Service Policy on Humane Care and Use of Laboratory Animals and were approved by the University of Kentucky's Institutional Animal Care and Use Committee. All procedures were conducted in a manner to minimize pain and discomfort of the animals. Young adult (9-10 week old) male and female C57BL/6J mice (RRID:IMSR_JAX:000664, Jackson Laboratories) were grouphoused for one week prior to experimentation and kept in a 12/12-hr light/dark cycle in a temperaturecontrolled vivarium room with free access to food and water. For the surgical procedures, animals ( $n=5$ /group) were randomly assigned to either sham surgery or controlled cortical impact (TBI) groups. All surgical procedures were performed using techniques published previously including post-surgical recovery (Mbye et al. 2008)

\section{Isolation of CD11b+ Cells from Bone Marrow and Brain}

Animals were sacrificed at $3 \mathrm{hr}, 24 \mathrm{hr}, 7$ days and 14 days following TBI. CD11b positive (CD11b+) cells were isolated from bone marrow and brain using EasySep ${ }^{\text {TM }}$ Mouse CD11b Positive Selection Kit II (Catalog \# 18970, STEMCELL Technologies) following the manufacturer's protocol. Briefly, bone marrow cells were harvested by flushing femurs and tibias with DMEM/F-12 with $10 \%$ FBS and $1 \%$ penicillin/streptomycin (Invitrogen Life Technologies) using syringe with a 25-gauge needle. A single cell suspension was prepared by passing harvested bone marrow cells through 18-gauge needle 4-6 times. After centrifugation at $300 \mathrm{Xg}$ for $5 \mathrm{~min}$, red blood cell (RBC) depletion was performed by adding $3 \mathrm{ml}$ RBC lysis buffer ( $0.15 \mathrm{M} \mathrm{NH}_{4} \mathrm{Cl}, 10 \mathrm{M} \mathrm{KHCO}_{3}$, and $0.1 \mathrm{M} \mathrm{Na}_{2} \mathrm{EDTA}, \mathrm{pH}$ 7. 4) on ice for $3 \mathrm{~min}$. RBC lysis was stopped by adding $15 \mathrm{ml}$ of EasySep ${ }^{\mathrm{TM}}$ Buffer (Catalog \#20144) followed by centrifugation at $300 \mathrm{Xg}$ for $5 \mathrm{~min}$. Cells were resuspended in $0.5 \mathrm{ml}$ EasySep $^{\text {TM }}$ Buffer and subjected to CD11 b+ cell isolation using EasySep ${ }^{\mathrm{TM}}$ Mouse CD11b Positive Selection Kit II.

For brain $\mathrm{CD} 11 \mathrm{~b}+$ cells isolation, the injured or surgery hemisphere was minced in a petri dish containing $1 \mathrm{ml}$ digestion media (DMEM/F-12, 15 mM HEPES, 2\% FBS, 20 units/ml Papain (Catalog \#07465) and 1 $\mathrm{mg} / \mathrm{ml}$ DNase I). Minced brain pieces were then transferred to $50 \mathrm{ml}$ conical tubes and incubated at $37^{\circ} \mathrm{C}$ for 20 min on a gently shaking platform. A single cell suspension was obtained by filtering the digested tissue through a $70 \mu \mathrm{m}$ nylon mesh strainer and the strainer rinsed with sample preparation media 
(DMEM/F-12, $15 \mathrm{mM}$ HEPES and 2\% FBS). The cell suspension was subjected to centrifugation at $300 \mathrm{Xg}$ for $10 \mathrm{~min}$ at $4^{\circ} \mathrm{C}$. After removing the supernatant, $6 \mathrm{ml}$ of $30 \%$ isotonic percoll solution (Catalog \#170891-01, GE Healthcare) was added to the suspended pellet followed by centrifugation at $700 \mathrm{Xg}$ for 10 min at $4^{\circ} \mathrm{C}$ with the brake off. The upper myelin layer was carefully removed and the pellet subjected to RBC depletion and CD11b+ cell isolation as above.

\section{RNA Isolation and Analysis}

Isolation and analysis of total RNAs from CD11b+ cells followed the procedures described previously (Wang et al. 2015; Wang et al. 2020). MiRNA/gene expression were analyzed using TaqMan assays (ThermoFisher Scientific). Small nuclear U6 RNA and Hprt (hypoxanthine phosphoribosyltransferase 1) were served as endogenous controls for normalizing miRNA and gene expression in the same sample, respectively.

\section{Statistical Analysis}

GraphPad Prism (Version 8.0.2; San Diego, CA, United States; RRID:SCR_002798) was used for statistical analyses. All data in this report presented as means \pm standard error of the mean (SEM). Repeatedmeasures two-way analysis of variance (ANOVA) was used for comparison among groups followed by Tukey's post hoc test to identify specific differences between groups. A value of $p<0.05$ with $95 \%$ confidence interval is considered statistically significant. Number of animals used in each study was represented in figure legends section of each result.

\section{Results}

\section{Preferential Enrichment of Inflammatory MiRNAs in CD11b + Cells from Brain and Bone Marrow}

TaqMan RT-qPCR was used to measure selected inflammatory miRNAs including miR-146a-5p, miR-223$3 p$, miR-155-5p, and miR-150-5p levels in brain and bone marrow CD11b + cells from female and male naïve mice. While miR-146a and miR-150 levels were detected in bone marrow CD11b + cells in both females and males, they were more than 25-fold higher in brain CD11b + cells compared to bone marrow in both sexes (Fig. 1). Both miRNAs showed higher levels in brain CD11b + cells of male mice compared to females, but only miR-150 reached statistical significance. Conversely, miR-223-3p, and miR-155-5p were more than 25 -fold higher in CD11b + cells isolated from bone marrow relative to brain in both sexes, with only miR-155-5p showing significant sex specific enrichment (males > females) in brain (Fig. 1). This pattern demonstrates that inflammatory miRNAs are differentially enriched in brain and bone marrow CD11b + cells.

Sex-specific Alterations of Inflammatory MiRNAs in CD11b + Cells Following Severe TBI 
To assess sex-specific miRNA alterations following TBI, we measured levels of these miRNA in CD11b + cells from injured cortical tissue and bone marrow at $3 \mathrm{hr}, 24 \mathrm{hr}, 7$ days, and 14 days after severe TBI. Levels of pro-inflammatory miR-155-5p in brain CD11b + cells were significantly increased in both female and male mice after $\mathrm{TBl}$, although females showed a significantly greater induction at $3 \mathrm{hr}$ compared to males (Fig. 2a). The levels of anti-inflammatory miR-146a were altered marginally over time (Fig. 2b), while miR-223-3p levels significantly increased by $24 \mathrm{hr}$ after TBI in both females and males, with females having significantly higher levels compared to males (Fig. 2c). MiR-150-5p was significantly increased in females but not males at 7 days and 14 days post-injury (Fig. 2d). Interestingly, we did not observe any TBI-related or sex differences in inflammatory miRNAs in bone marrow CD11b + cells (Suppl Fig. 1).

\section{Sex-specific Alterations of Brain MiR-223-3p Targets and Inflammatory Gene Expressions in CD11b + Cells Following Severe TBI}

We then examined the expression profile of miR-223-3p specific targets and related inflammatory genes in brain CD11b + cells of female and male mice following TBI. The sham animals did not exhibit any sexspecific patterns in the expression levels of any of the inflammatory genes examined. Reverse-correlated with the increase in miR-223-3p reported above (Fig. 2C), we found that the levels of two miR-223-3p targets, FBXW7 and TRAF6, significantly decreased at $24 \mathrm{hr}$ post-injury only in brain CD $11 \mathrm{~b}+$ cells from female mice (Fig. 3a). Interestingly, we observed an increased expression of the anti-inflammatory gene, $A R G 1$, in both female and male injured mice compare to sham animals at $24 \mathrm{hr}$ post-injury, with females showing significantly higher expression than males (Fig. 3b). At this same $24 \mathrm{hr}$ time point, brain CD11b + cells from female, but not male, mice had significantly higher expression of another anti-inflammatory gene, IL4 (Fig. 3b).

Several pro-inflammatory gene markers also exhibited a sex-related differential expression in brain CD11b + cells following TBI. For example, CCL2 was significantly increased in both female and male mice after TBI, although significantly greater increase was observed in males relative to females at $24 \mathrm{hr}$ (Fig. 3c). In addition, there was a significant increase in COX2 expression only in male mice at $3 \mathrm{hr}$ and 24 $\mathrm{hr}$, which gradually decreased with time (Fig. 3c). The levels of TNFa and $I L 1 b$ showed different sexrelated expression patterns over time following injury (Fig. 3c). Specifically, both pro-inflammatory markers peaked at $3 \mathrm{hr}$ after TBI in males and at $24 \mathrm{hr}$ in females, with significantly higher levels in females compared to males at $24 \mathrm{hr}$ (Fig. 3c).

\section{Discussion}

Our present study demonstrates the existence of a significant and previously unreported preferential enrichment and sex-specific response of inflammatory miRNAs in brain and bone marrow CD11b + cells. The inflammatory miRNAs examined in this study play key roles in inflammatory signaling in myeloid cells and are all implicated in TBI (Liu et al. 2014; Redell et al. 2009; Wang et al. 2015): miR-146a is a negative regulator of NF-KB pathway (Taganov et al. 2006); miR-223-3p plays a key role in limiting 
myeloid cell pro-inflammatory signaling (Jiao et al. 2021); miR-155-5p is a highly inducible, proinflammatory miRNA (Jablonski et al. 2016; Sun et al. 2012); and miR-150-5p is an important modulator for differentiation and activation of immune cells (Zhou et al. 2007). Our results revealed that miR-146a$5 p$ and miR-150-5p are relatively enriched in brain CD11b + resident microglia and that miR-223-3p and miR-155-5p are relatively enriched in bone marrow CD11b + monocytes and macrophages. Although we did not detect significant changes of these miRNAs in bone marrow CD11b + cells following TBI, intriguingly, the response of brain CD11b + miRNAs to TBI are vastly different with regards to sex and time following injury. For example, an early and significant elevation of pro-inflammatory miR-155-5p in brain CD11b + cells was observed in both female and male mice compared to shams, which returned to sham levels by 7 days post-injury but again increased significantly at 14 days. It is interesting to point out that the levels of miR-155-5p were higher in brain CD11b + cells from naïve males, while the levels of miR155-5p in CD11b + cells from injured female brain were significantly higher than males at the earliest time point examined ( $3 \mathrm{hr}$ ). Anti-inflammatory miR-223-3p was significantly increased in brain CD11b + cells from males at $3 \mathrm{hr}$, and in both sexes at $24 \mathrm{hr}$, with significant higher levels in females compared to males. However, this increase diminished at 7 and 14 days following TBI in both females and males. The dynamic changes of pro- and anti-inflammatory miRNAs is consistent with the experimental and clinical observations that while reparative anti-inflammatory signaling peak shortly following brain injury, proinflammatory effectors persist for weeks to months to years (Loane et al. 2014; Pierce et al. 1998).

We further examined the expression of targets of miR-223-3p and several inflammatory marker genes. MiR-223-3p is directly associated with reparative inflammatory response via regulation of key inflammatory signaling pathways such as NF-KB and the inflammasome (Bauernfeind et al. 2012; Kumar et al. 2014; Zhou et al. 2018). Our data showed a significant down regulation of the miR-223-3p validated targets FBXW7 and TRAF6, which are key modulators of the NF-KB pathway, in females compared to males at $24 \mathrm{hr}$ following TBI. Interestingly, the expression of the anti-inflammatory genes, ARG1 and IL4 were significantly increased in female relative to male mice and sham at the same $24 \mathrm{hr}$ time point. Whether the increased expression of anti-inflammatory genes is a direct result of miR-223-3p regulation will need to be further investigated. The pro-inflammatory marker gene profiles were more diverse. There is a clear increase in the levels of the pro-inflammatory genes CCL2, COX2, TNFa, and IL $1 \mathrm{~b}$ in both male and female injured brains compared to sham. However, COX2 and CCL2 were higher in males at 3 and 24 hr post-injury, whereas greater levels of $T N F a$ and $I L 1 b$ were observed in females TBI at $24 \mathrm{hr}$, although an initial higher level were seen in males at $3 \mathrm{hr}$. These complicated sex-bias inflammatory responses could be a consequence of multiple signaling events that involve the dynamic changes of inflammatory miRNAs. Regardless, these results are in line with the findings that inflammatory factors display sexual dimorphic responses following TBI (Spani et al. 2018; Villapol et al. 2017).

We realize there are limitations and caveats of the current study. For example, there are many other inflammatory responsive miRNAs that may participate in cell/tissue- and sexual-specific modulation of inflammatory response, but our studies were limited to only four well-studied inflammatory responsive miRNAs. Moreover, we only examined two acute time points ( 3 and $24 \mathrm{hr}$ ) and two chronic time points (7 and $14 \mathrm{~d}$ ). We believe there could be additional significant sex-related changes at other time intervals. In 
addition, although CD11b + cells contain major monocytes, such as macrophages and microglia, the isolation procedure used cannot distinguish subtypes of these monocytes. Future investigations will be required to fully address the tissue/cell specific and sexual dimorphic miRNA inflammatory response following TBI.

\section{Declarations}

Funding: This work is supported by a grant (18-8A) from the Kentucky Spinal Cord and Head Injury Research Trust to JES and WXW.

Conflicts of interest/Competing interests: The authors have no conflicts of interest to declare that are relevant to the content of this article.

Availability of data and material: Not applicable

Code availability: Not applicable

Authors' contributions: Study concept and design: JES, WXW, PP; Experimental implementations: PP, WXW, SP, UG; Data acquisition and analysis: PP, WXW; Statistical analysis: PP, WXW; Manuscript preparation: PP, WXW, JES; Manuscript editing and review: JES, WXW, PP, SP, UG

Compliance with Ethical Standards: All animal procedures used in this study conformed to the National Institutes of Health Guide for the Care and Use of Laboratory Animals and were approved by the University of Kentucky's Institutional Animal Care and Use Committee (IACUC protocol no.: 2019-3223).

Ethics approval (include appropriate approvals or waivers): Not applicable

Consent to participate (include appropriate statements): Not applicable

Consent for publication (include appropriate statements): Not applicable

\section{References}

1. Acaz-Fonseca E, Duran JC, Carrero P, Garcia-Segura LM, Arevalo MA (2015) Sex differences in glia reactivity after cortical brain injury. Glia 63(11):1966-1981. doi:10.1002/glia.22867

2. Bauernfeind F, Rieger A, Schildberg FA, Knolle PA, Schmid-Burgk JL, Hornung V (2012) NLRP3 inflammasome activity is negatively controlled by miR-223. J Immunol 189(8):4175-4181. doi:10.4049/jimmunol.1201516

3. Cardoso AL, Guedes JR, Pereira de Almeida L, Pedroso de Lima MC (2012) miR-155 modulates microglia-mediated immune response by down-regulating SOCS-1 and promoting cytokine and nitric oxide production. Immunology 135(1):73-88. doi:10.1111/j.1365-2567.2011.03514.x

4. Doran SJ, Ritzel RM, Glaser EP, Henry RJ, Faden Al, Loane DJ (2019) Sex Differences in Acute Neuroinflammation after Experimental Traumatic Brain Injury Are Mediated by Infiltrating Myeloid 
Cells. J Neurotrauma 36(7):1040-1053. doi:10.1089/neu.2018.6019

5. Gordon S, Taylor PR (2005) Monocyte and macrophage heterogeneity. Nat Rev Immunol 5(12):953964. doi:10.1038/nri1733

6. Jablonski KA, Gaudet AD, Amici SA, Popovich PG, Guerau-de-Arellano M (2016) Control of the Inflammatory Macrophage Transcriptional Signature by miR-155. PLoS One 11(7):e0159724. doi:10.1371/journal.pone.0159724

7. Jiao P, Wang XP, Luoreng ZM, Yang J, Jia L, Ma Y, Wei DW (2021) miR-223: An Effective Regulator of Immune Cell Differentiation and Inflammation. Int J Biol Sci 17(9):2308-2322. doi:10.7150/ijbs. 59876

8. Jin X, Ishii H, Bai Z, Itokazu T, Yamashita T (2012) Temporal changes in cell marker expression and cellular infiltration in a controlled cortical impact model in adult male C57BL/6 mice. PLoS One 7(7):e41892. doi:10.1371/journal.pone.0041892

9. Johnnidis JB, Harris MH, Wheeler RT, Stehling-Sun S, Lam MH, Kirak O, Brummelkamp TR, Fleming MD, Camargo FD (2008) Regulation of progenitor cell proliferation and granulocyte function by microRNA-223. Nature 451(7182):1125-1129. doi:10.1038/nature06607

10. Johnson VE, Stewart JE, Begbie FD, Trojanowski JQ, Smith DH, Stewart W (2013) Inflammation and white matter degeneration persist for years after a single traumatic brain injury. Brain 136(Pt 1):2842. doi:10.1093/brain/aws322

11. Kodama L, Guzman E, Etchegaray JI, Li Y, Sayed FA, Zhou L, Zhou Y, Zhan L, Le D, Udeochu JC, Clelland CD, Cheng Z, Yu G, Li Q, Kosik KS, Gan L (2020) Microglial microRNAs mediate sex-specific responses to tau pathology. Nat Neurosci 23(2):167-171. doi:10.1038/s41593-019-0560-7

12. Kumar A, Alvarez-Croda DM, Stoica BA, Faden Al, Loane DJ (2016) Microglial/Macrophage Polarization Dynamics following Traumatic Brain Injury. J Neurotrauma 33(19):1732-1750. doi:10.1089/neu.2015.4268

13. Kumar V, Palermo R, Talora C, Campese AF, Checquolo S, Bellavia D, Tottone L, Testa G, Miele E, Indraccolo S, Amadori A, Ferretti E, Gulino A, Vacca A, Screpanti I (2014) Notch and NF-kB signaling pathways regulate miR-223/FBXW7 axis in T-cell acute lymphoblastic leukemia. Leukemia 28(12):2324-2335. doi:10.1038/leu.2014.133

14. Liu L, Sun T, Liu Z, Chen X, Zhao L, Qu G, Li Q (2014) Traumatic brain injury dysregulates microRNAs to modulate cell signaling in rat hippocampus. PLoS One 9(8):e103948.

doi:10.1371/journal.pone.0103948

15. Liu NK, Xu XM (2011) MicroRNA in central nervous system trauma and degenerative disorders. Physiol Genomics 43(10):571-580. doi:10.1152/physiolgenomics.00168.2010

16. Loane DJ, Kumar A, Stoica BA, Cabatbat R, Faden Al (2014) Progressive neurodegeneration after experimental brain trauma: association with chronic microglial activation. J Neuropathol Exp Neurol 73(1):14-29. doi:10.1097/nen.0000000000000021

17. Mbye LH, Singh IN, Sullivan PG, Springer JE, Hall ED (2008) Attenuation of acute mitochondrial dysfunction after traumatic brain injury in mice by NIM811, a non-immunosuppressive cyclosporin A 
analog. Exp Neurol 209(1):243-253. doi:10.1016/j.expneurol.2007.09.025

18. Morganti JM, Riparip LK, Rosi S (2016) Call Off the Dog(ma): M1/M2 Polarization Is Concurrent following Traumatic Brain Injury. PLoS One 11(1):e0148001. doi:10.1371/journal.pone.0148001

19. Pierce JE, Smith DH, Trojanowski JQ, Mclntosh TK (1998) Enduring cognitive, neurobehavioral and histopathological changes persist for up to one year following severe experimental brain injury in rats. Neuroscience 87(2):359-369. doi:10.1016/s0306-4522(98)00142-0

20. Redell JB, Liu Y, Dash PK (2009) Traumatic brain injury alters expression of hippocampal microRNAs: potential regulators of multiple pathophysiological processes. J Neurosci Res 87(6):1435-1448. doi:10.1002/jnr.21945

21. Shakerian L, Ghorbani S, Talebi F, Noorbakhsh F (2018) MicroRNA-150 targets PU.1 and regulates macrophage differentiation and function in experimental autoimmune encephalomyelitis. $J$ Neuroimmunol 323:167-174. doi:10.1016/j.jneuroim.2018.06.010

22. Sharma S, Eghbali M (2014) Influence of sex differences on microRNA gene regulation in disease. Biol Sex Differ 5(1):3. doi:10.1186/2042-6410-5-3

23. Spani CB, Braun DJ, Van Eldik LJ (2018) Sex-related responses after traumatic brain injury: Considerations for preclinical modeling. Front Neuroendocrinol 50:52-66. doi:10.1016/j.yfrne.2018.03.006

24. Sun Y, Cai J, Ma F, Lu P, Huang H, Zhou J (2012) miR-155 mediates suppressive effect of progesterone on TLR3, TLR4-triggered immune response. Immunol Lett 146(1-2):25-30. doi:10.1016/j.imlet.2012.04.007

25. Taganov KD, Boldin MP, Chang KJ, Baltimore D (2006) NF-kappaB-dependent induction of microRNA miR-146, an inhibitor targeted to signaling proteins of innate immune responses. Proc Natl Acad Sci U S A 103(33):12481-12486. doi:10.1073/pnas.0605298103

26. Villapol S, Loane DJ, Burns MP (2017) Sexual dimorphism in the inflammatory response to traumatic brain injury. Glia 65(9):1423-1438. doi:10.1002/glia.23171

27. Wang WX, Prajapati P, Nelson PT, Springer JE (2020) The Mitochondria-Associated ER Membranes Are Novel Subcellular Locations Enriched for Inflammatory-Responsive MicroRNAs. Mol Neurobiol 57(7):2996-3013. doi:10.1007/s12035-020-01937-y

28. Wang WX, Visavadiya NP, Pandya JD, Nelson PT, Sullivan PG, Springer JE (2015) Mitochondriaassociated microRNAs in rat hippocampus following traumatic brain injury. Exp Neurol 265:84-93. doi:10.1016/j.expneurol.2014.12.018

29. Zhao JL, Rao DS, Boldin MP, Taganov KD, O'Connell RM, Baltimore D (2011) NF-kappaB dysregulation in microRNA-146a-deficient mice drives the development of myeloid malignancies. Proc Natl Acad Sci U S A 108(22):9184-9189. doi:10.1073/pnas.1105398108

30. Zhou B, Wang S, Mayr C, Bartel DP, Lodish HF (2007) miR-150, a microRNA expressed in mature B and T cells, blocks early B cell development when expressed prematurely. Proc Natl Acad Sci U S A 104(17):7080-7085. doi:10.1073/pnas.0702409104 
31. Zhou W, Pal AS, Hsu AY, Gurol T, Zhu X, Wirbisky-Hershberger SE, Freeman JL, Kasinski AL, Deng Q (2018) MicroRNA-223 Suppresses the Canonical NF-kB Pathway in Basal Keratinocytes to Dampen Neutrophilic Inflammation. Cell Rep 22(7):1810-1823. doi:10.1016/j.celrep.2018.01.058

\section{Figures}

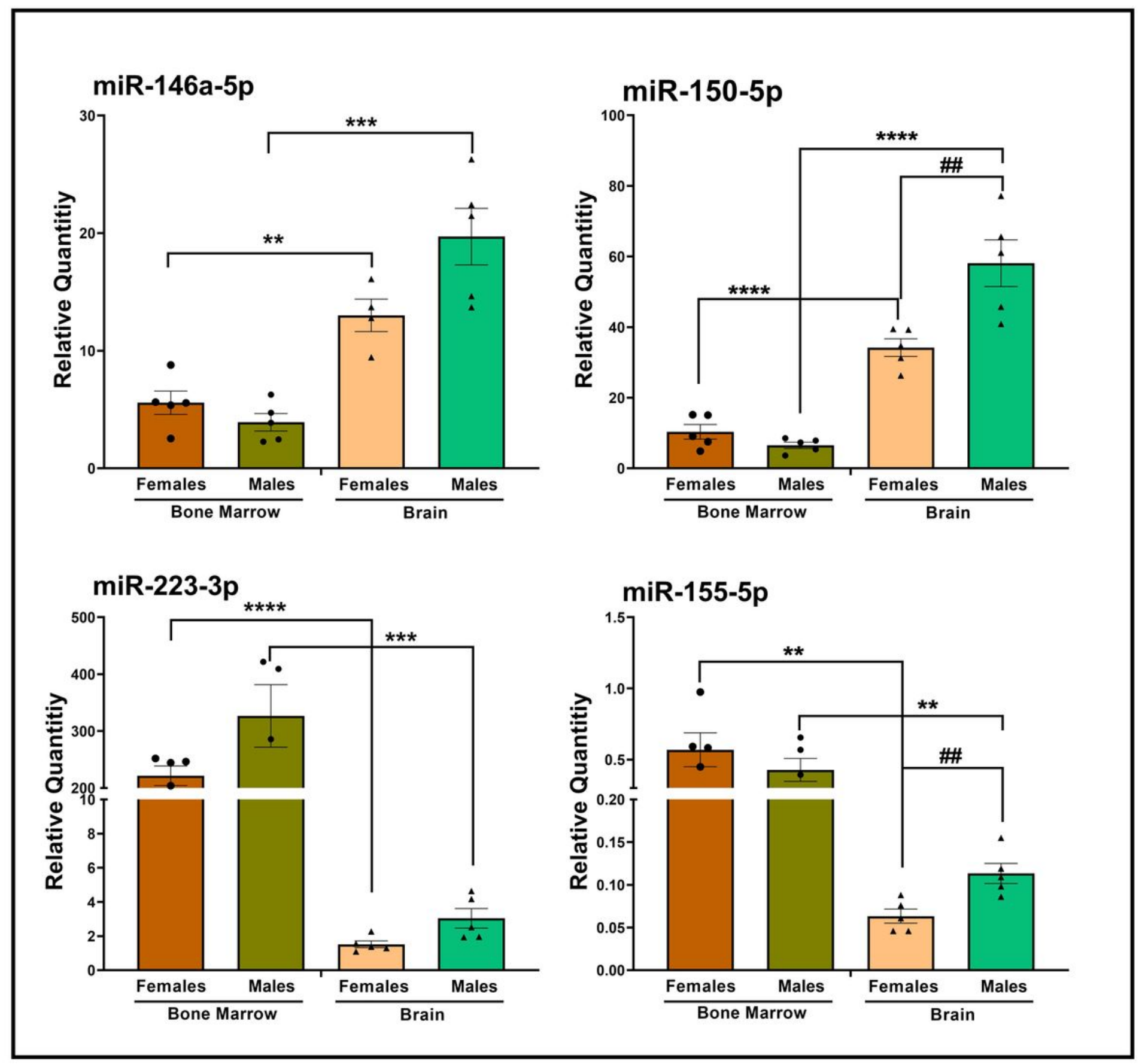

Figure 1

Enrichment of miRNAs in CD11b+ cells of naïve mice. Levels of inflammatory miRNAs miR-146a-5p and miR-150-5p, miR-223-3p, and miR-155-5p in brain and bone marrow CD11b+ cells of male and female 
mice. " $\star$ " indicate significant difference between bone marrow and brain and "\#" indicate significant difference between male and female ( $n=5$ mice/per group)

a

miR-155-5p

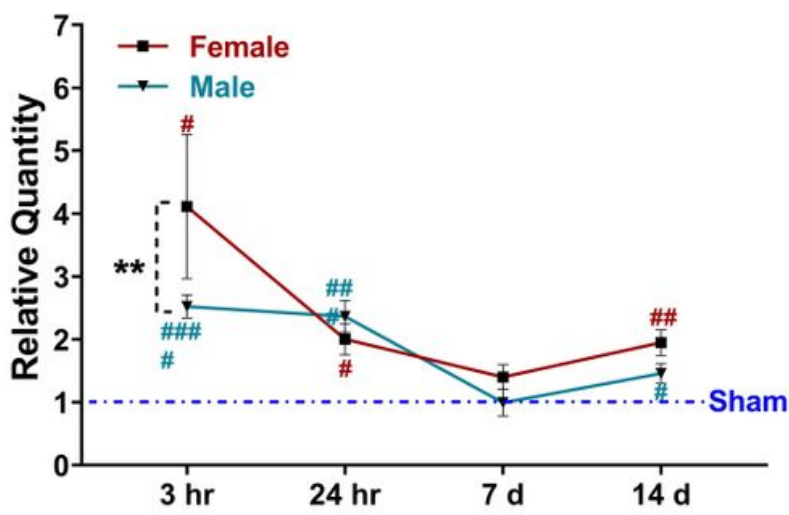

C

miR-223-3p

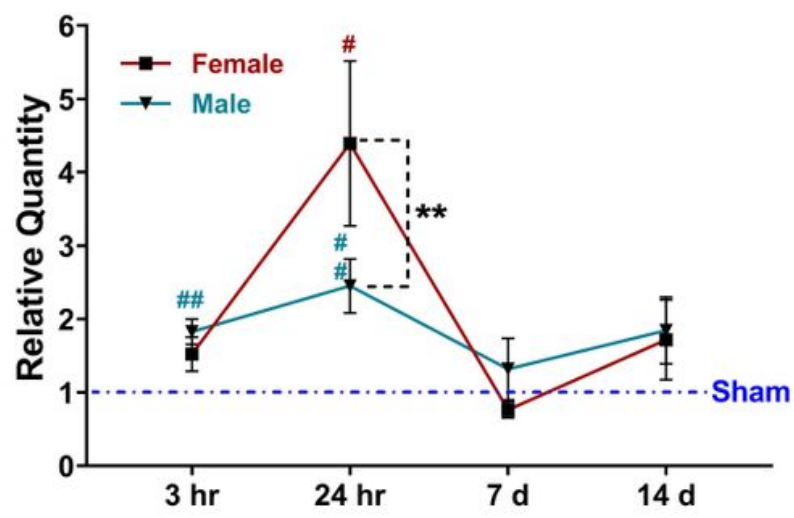

b

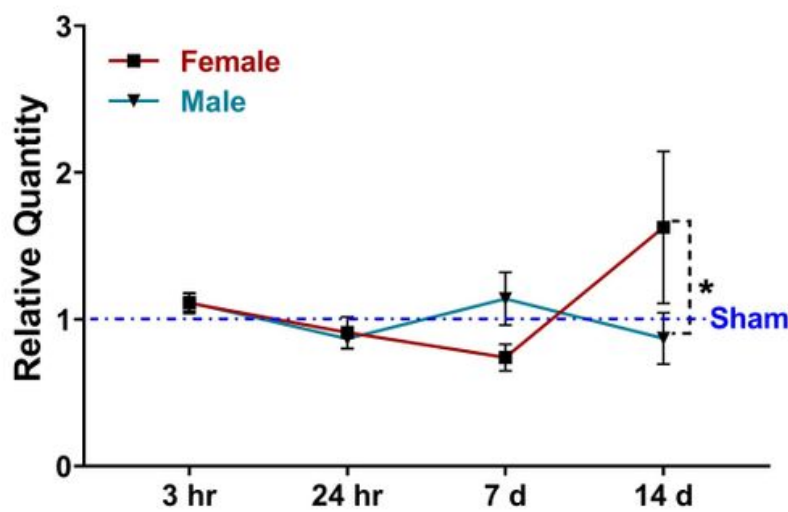

d

$\operatorname{miR}-150-5 p$

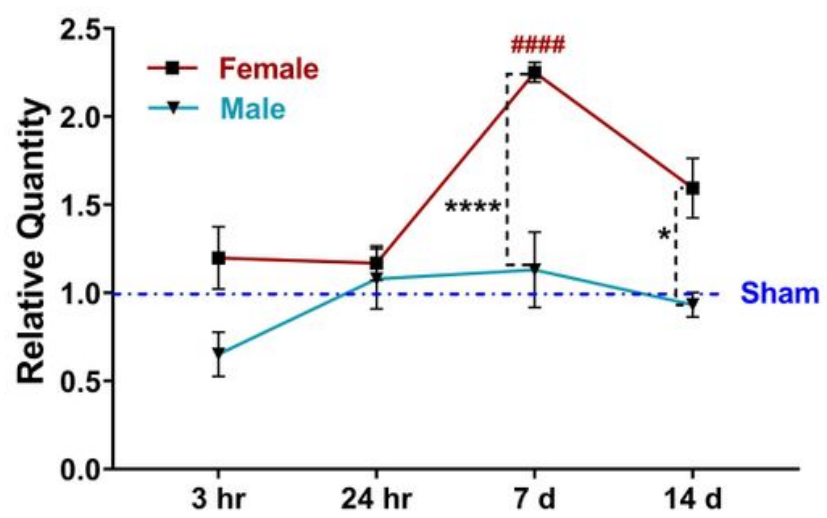

\section{Figure 2}

Response of inflammatory miRNAs to TBI over time. Levels of inflammatory miRNAs (a) miR-155, (b) miR-146a, (c) miR-223a, and (d) miR-150 in brain CD11b+ cells of male and female mice at different time points following TBI. Levels of sham group were set as 1. "\#” indicate significant difference between sham and TBI group and " $\star$ " indicate significant difference between females and males ( $n=4-8$ mice/time point) 


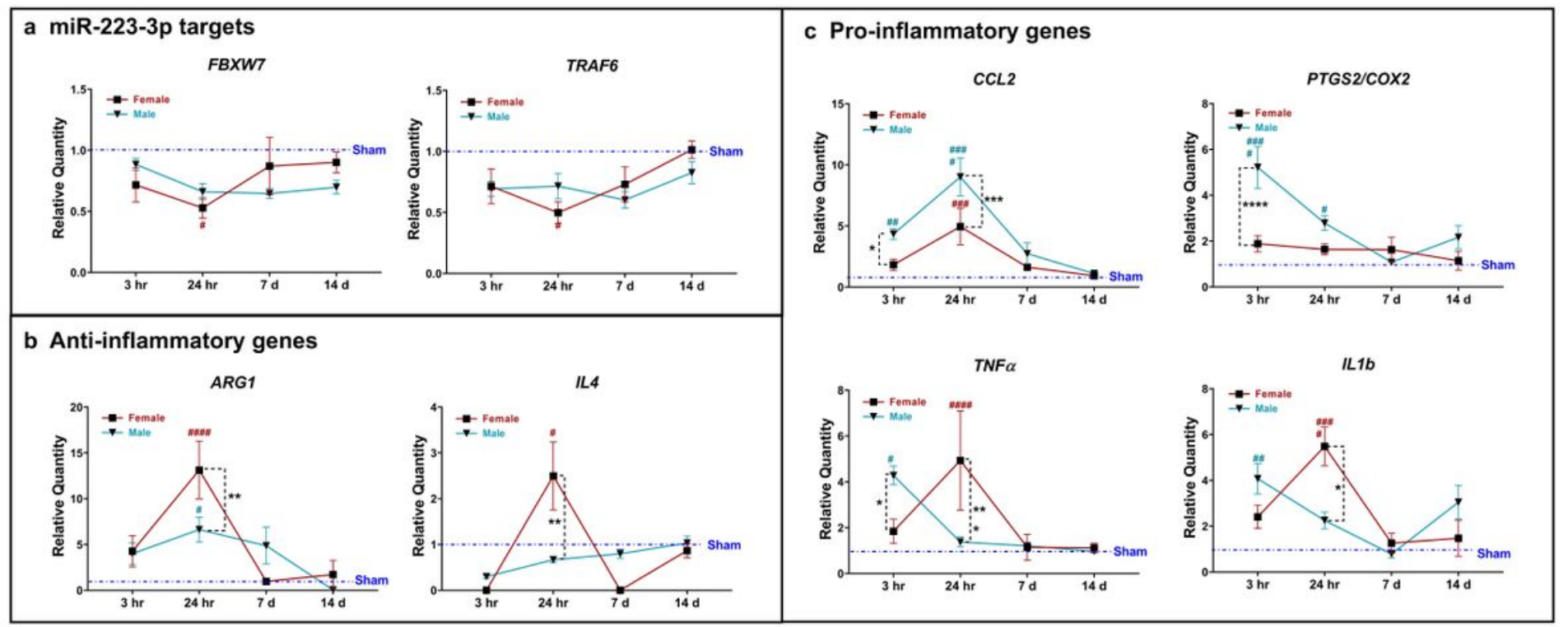

\section{Figure 3}

Changes of inflammatory marker genes and targets of miR-223-3p following TBI. Targets of miR-223-3p (a), anti-inflammatory marker genes (b), and pro-inflammatory marker genes (c) in brain CD11b+ cells of male and female mice at different time points following TBI. Levels of sham group were set as 1. "\#" indicate significant difference between sham and TBI group and "*" indicate significant difference between females and males ( $n=4-8$ mice/time point)

\section{Supplementary Files}

This is a list of supplementary files associated with this preprint. Click to download.

- SupplFig1.jpg 